ISSN: $1130-3743$

\title{
APROXIMACIÓN A UN PLANTEAMIENTO SISTEMÁTICO DE LA EDUCACIÓN NO FORMAL: LA PERSONA COMO CENTRO DE DESARROLLO
}

\author{
Approximation to systematic position of nonformal \\ education: person as the development centre
}

Rafael SÁEZ ALONSO y Carolina FERNÁNDEZ-SALINERO MIGUEL

Departamento de Teoria e Historia de la Educación. Facultad de Educación. Centro de Formación del Profesorado. Universidad Complutense de Madrid. $P^{o}$ Rector Royo Villanova, $s / n$. 28040 Madrid

BIBLID [(1130-3743) 10, 1998, 169-187]

RESUMEN

El significado y la utilidad de la educación no formal en el momento presente nos fuerza a investigar y reconocer sus posibilidades y a incidir en su desarrollo en virtud de las personas más que en la tipología de programas, acciones e instituciones. Es la persona la que se educa y/o es educada. Nuestra reflexión parte de la Teoría General de Sistemas. La educación es un sistema de elementos en interacción mutua y continua. La educación no formal es un sistema abierto y genera una serie de procesos de comunicación para lograr efectos educativos. Es la totalidad de la vida la que va generando el proceso de educación de la persona. Intentamos aproximarnos a un concepto de educación no formal que se define dentro de la estructura sistémica de educación.

\section{SUMMARY}

The meaning and the usefulness of the nonformal education in the present moment force us to investigate and recognize its possibilities ant to impact in its development through the persons, more than the type of programs, actions and institutions. The person is who is educated. Our reflection parts of the General System Theory. The education is a system of elements in mutual and continuous interac- 
tion. The nonformal education is an open system and generates different communications process to achieve educational effects. The life is going to generate the personal educational process. We attempt to approximate to a nonformal education concept that it is defined within the systemic education structure.

\section{Planteamientos introductorios}

La educación no formal se constituye, en el momento presente, en una vía de desarrollo personal y social que trasciende la estructura educativa, académica y escolar, propiciando un aprendizaje más próximo a las necesidades de los individuos y completando la enseñanza institucionalizada de los centros formalizados de estudio. Es, además, un instrumento que favorece la igualdad y el acceso a la cultura a una gran diversidad de "clientes", algunos de ellos alejados de la misma, bien por su ubicación espacial, bien por su situación socioeconómica o por sus dificultades de normalización educativa.

Esta visión, altamente positiva, del significado y utilidad de la educación no formal nos fuerza a investigar sobre el término, a reconocer sus posibilidades y a incidir en su desarrollo en virtud de las personas más que en la tipología de programas, acciones e instituciones constituidas a su sombra. Es la persona la que se educa y/o es educada. Es el ser humano el que, por medio de la educación, debe desarrollar todas sus capacidades. Como afirma Delors (1996), la educación:

Ha de ayudar a superar las tensiones entre lo mundial y lo local, lo universal y lo singular, la tradición y la modernidad, el largo y el corto plazo, la indispensable competencia y la preocupación por la igualdad de oportunidades, el extraordinario desarrollo de los conocimientos y las capacidades de asimilación del ser humano, lo espiritual y lo material (pp. 17-18).

Es la persona la sede donde vive, se desarrolla y crece la educación. El tesoro de la educación está destinado a ser descubierto por la persona a través de una búsqueda sistemática y original, deliberada y creativa en cada época y contexto. Habrá momentos o períodos de tiempo en los cuales lo que acontece en la educación se limitará al ámbito institucional y otras veces, las experiencias educativas tendrán lugar "antes, después, y fuera de los marcos formales de las instituciones" (Sancho y Hernández, 1997, p. 96).

A través de estas ideas pretendemos ofrecer una reflexión sobre la excesiva parcialización y, a veces enfrentamiento, entre la educación no formal y otros planteamientos educativos (educación formal y educación informal). Para ello, nos vamos a apoyar en los autores que han investigado sobre este tema y en las aportaciones derivadas de la Teoría General de Sistemas, instrumentos ambos que nos van a permitir conocer el estado de la cuestión e identificar así las peculiaridades que el amplio marco de la extensión del fenómeno educativo evidencia, por medio de su aproximación a ámbitos de desarrollo no tan marcadamente conformados como respuesta estructurada que emana del poder administrativo, sino más bien, identificados como posibilidades de mejora personal e implicación social. 
RAFAEL SÁEZ ALONSO Y CAROLINA FERNÁNDEZ-SALINERO MIGUEL

2. ANÁLISIS DE LAS DEFINICIONES MÁS SIGNIFICATIVAS DE EDUCACIÓN NO FORMAL

La literatura consultada en relación con la educación no formal, nos aporta luz para acercarnos a la misma a través de las diversas definiciones que autores representativos de la reflexión pedagógica han generado en las últimas décadas. En este sentido, a continuación recogemos las más significativas -por orden cronológico- como punto de partida para nuestra aportación posterior.

"Toda actividad organizada, sistemática, educativa, realizada fuera del marco del sistema oficial, para facilitar determinadas clases de aprendizajes en subgrupos particulares de la población, tanto adultos como niños" (Coombs y Ahmed, 1975, pp. 6-28).

"Parte de la constatación de la infrautilización del potencial de la educación informal y no formal, principalmente porque el medio no favorece, sino excepcionalmente, la autoinstrucción o iniciativa formativa individual. Ante este panorama, resulta imprescindible impulsar medidas formativas que se desenvuelvan dentro de estas dos vertientes e insistir sobre la necesidad de vincular sistemáticamente el ejercicio de una profesión a la formación permanente" (Furter, 1976).

"La educación no formal se esboza simplemente como un conjunto de actividades educativas no claramente organizadas, dirigidas a una clientela indefinida, con programas de tiempo parcial que tiene la función de complementar la enseñanza formal o de crear una segunda oportunidad para aquellos que no ban podido asistir a la enseñanza formal. (Coombs, 1978, pp. 202-203).

"La expresión educación no formal se acuña para satisfacer la necesidad de respuestas extraescolares a demandas nuevas y diferentes de las que atiende ordinariamente el sistema educativo" (La Belle, 1982, p. 160).

"La educación no formal se legitima por las posibilidades que ofrece para superar las inevitables estratificaciones de la escolaridad que, en la mayoría de las sociedades existentes, guarda estrecho vinculo con la estratificación social. De este modo la educación no formal brinda acceso a una democratización educativa más efectiva, superadora de gradaciones preestablecidas y en la que la participación tiene todos los rasgos de un instrumento formativo" (Nassif, 1983, pp. 339-340).

"La educación no formal es el proceso de adquisición y el conjunto de competencias, destrezas y actitudes educativas adquiridas con estimulos directamente educativos en actividades no conformadas por el sistema escolar" (Touriñán, 1984, p. 129).

"Actividades o programas organizados fuera del sistema escolar establecido, pero dirigidos sin embargo bacia objetivos concretos de educación" (Tesauro de la Educación/Unesco-OIE, 1984, p. 202). 
"Educación no formal es todo proceso de aprendizaje que se acomete a lo largo de la vida para el acceso efectivo a conocimientos y destrezas, básicos y avanzados, esté o no institucionalizado y se obtengan o no certificados y acreditaciones al final del mismo, pero que permita asumir eficazmente responsabilidades concretas en la vida activa" (Díez Hochleitner, 1991, p. 54).

"Educación no formal significa en la práctica "educación no registrada" 0 , lo que es lo mismo, acciones educativas individuales o colectivas que se llevan a cabo sin inscripciones o registros oficiales y de las que, en consecuencia, no se tiene constancia oficial alguna" (García Garrido, 1991, p. 54).

"Educación no formal es el conjunto de procesos, medios e instituciones específicas y diferenciadamente diseñados en función de explicitos objetivos de formación o de instrucción, que no están directamente dirigidos a la provisión de los grados propios del sistema educativo reglado" (Trilla, 1992, p. 28).

"Programas de educación no formal significa programas de intencionalidad explicitamente educativa, que poseen planificación previa, con vistas a lograr metas y objetivos que no pertenecen a los curricula escolares de los diversos niveles del sistema educativo" (Sarramona, Vázquez y Ucar, 1992, p. 92).

Estas definiciones demuestran la preocupación por este tema de numerosos autores, entre los que también cabe recordar a aquellos otros que han investigado sobre el mismo, recogiendo para ello las aportaciones de los precedentes. Entre esos autores hemos de mencionar los siguientes:

- Faure, 1973, pp. 266-267.

- Castillejo, 1987, pp. 31-102.

- García Carrasco, 1988, pp. 127-155.

- Radcliffe y Colletta, 1989, pp. 1.837-1.842.

- Srinivasan, 1989, pp. 1.852-1.855.

- $\quad$ Lowe, 1989, pp. 1.844-1.847.

- Quintana Cabanas, 1991, pp. 44-61.

- Colom, 1992, pp. 51-73.

- Núñez Cubero, 1993, pp. 207-234.

- Fermoso, 1994, pp. 109-125.

- Requejo, 1994, pp. 61-76.

- Vázquez, 1995, pp. 31-41.

- Sarramona, Vázquez y Colom, 1998, pp. 11-25.

La información documental analizada nos permite realizar el siguiente cuadroresumen sobre los criterios identificativos de la educación no formal (ver cuadro 1). Nos apoyamos para ello en los autores mencionados y en sus definiciones, desglosando de éstas los elementos que conceptualizan nuestro objeto de análisis y favorecen, a este respecto, una aproximación personal emanada de las ideas anteriormente señaladas. 
RAFAEL SÁEZ ALONSO Y CAROLINA FERNÁNDEZ-SALINERO MIGUEL APROXIMACIÓN A UN PLANTEAMIENTO SISTEMÁTICO DE LA EDUCACIÓN...

CUADRo 1. Cuadro-Resumen de definiciones de educación no formal

\begin{tabular}{|c|c|c|c|c|c|c|c|c|c|c|c|}
\hline AUTORES & $\begin{array}{l}\text { COOMBS } \\
\text { Y AHMED }\end{array}$ & COOMBS & NASSIF & TOURIÑ́́N & UNESCO & $\begin{array}{c}\text { DÍEZ } \\
\text { HOCHIEITNER }\end{array}$ & $\begin{array}{l}\text { GARCíA } \\
\text { GARRIDO }\end{array}$ & TRULA & $\begin{array}{l}\text { SARRAMONA } \\
\text { VÁZQUEZ Y UCAR }\end{array}$ & LA BELEE & FURTER \\
\hline ACREDITACIÓN & & & & & & X & & $\mathrm{X}$ & & & \\
\hline APRENDIZAJE & $\mathrm{X}$ & & & $\mathrm{X}$ & & $\mathrm{X}$ & & & & & $X$ \\
\hline CLIENTELA & $\mathrm{X}$ & $X$ & $\mathrm{X}$ & & & & $X$ & & & $\mathrm{X}$ & $X$ \\
\hline $\begin{array}{l}\text { COMPETENCIAS/ } \\
\text { DESTREZAS/ } \\
\text { ACTITUDES }\end{array}$ & & & & $X$ & & $\mathrm{X}$ & & & & & \\
\hline $\begin{array}{l}\text { COMPLEMENTO } \\
\text { FORMATIVO }\end{array}$ & $\mathrm{X}$ & $\mathrm{X}$ & $\mathrm{X}$ & $\mathrm{X}$ & & & & $\mathrm{X}$ & & $\mathrm{X}$ & \\
\hline DESCENTRALIZACIÓN & $\mathrm{X}$ & & $\mathrm{X}$ & $X$ & $\mathrm{X}$ & & $\mathrm{X}$ & $\mathrm{X}$ & & $\mathrm{X}$ & $\mathrm{X}$ \\
\hline EDUCACIÓN & $\mathrm{X}$ & $X$ & $\mathrm{X}$ & $\mathrm{X}$ & $\mathrm{X}$ & $X$ & $\mathrm{X}$ & $\mathrm{X}$ & $\mathrm{X}$ & $\mathrm{X}$ & $\mathrm{X}$ \\
\hline FLEXIBILDAD & & $X$ & $X$ & & & & & & & $\mathrm{X}$ & $\mathrm{X}$ \\
\hline institucionalzación & & & $X$ & & & $X$ & & $\mathrm{X}$ & & & \\
\hline INTENCIONALIDAD & $\mathrm{X}$ & $\mathrm{X}$ & $\mathrm{X}$ & $\mathrm{X}$ & $\mathrm{X}$ & $\mathrm{X}$ & $\mathrm{X}$ & $\mathrm{X}$ & $\mathrm{X}$ & $X$ & $\mathrm{X}$ \\
\hline $\begin{array}{l}\text { METAS } \\
\text { FORMATIVAS }\end{array}$ & & & $X$ & & $X$ & & & $X$ & $\mathrm{X}$ & $\mathrm{X}$ & $X$ \\
\hline $\begin{array}{l}\text { PROCESO } \\
\text { ORGANIZADO }\end{array}$ & $X$ & $X$ & $X$ & $X$ & $\mathrm{X}$ & $X$ & $\mathrm{X}$ & $\mathrm{X}$ & $X$ & $\mathrm{X}$ & $\mathrm{X}$ \\
\hline PROGRAMACIÓN & & $\mathrm{X}$ & & & $\mathrm{X}$ & & & & $\mathrm{X}$ & & \\
\hline SISTEMATIZACIÓN & $\mathrm{X}$ & & & $\mathrm{X}$ & & & & $X$ & & & $X$ \\
\hline
\end{tabular}

Cuadro elaboración propia

Si nos detenemos brevemente en el análisis del cuadro 1 , saltan a la vista algunas características que la mayoría de los autores destacan. En primer lugar y, como es obvio, la educación no formal es educación, es decir, es desarrollo perfectivo y armónico de la persona. Posee intencionalidad, esto es, se educa para conseguir una mejora de la persona; una persona educada es distinta a esta misma persona antes de vivir el proceso de educación. Y esta educación exige un pro- 
ceso organizado y realizado por personas preparadas y competentes en su rol; un proceso sistemáticamente planificado, pues como afirma Colom (1998) "no puede existir educación no formal sin planificar" (p. 165). Se programa con propósitos y objetivos claramente educativos.

Las otras características dan un tinte de no formal al paño de la educación. Existe aprendizaje, aunque no esté acreditado institucionalmente en la mayoría de los casos, para una clientela que pretende desarrollar competencias, destrezas y actitudes como un complemento formativo, con flexibilidad y alejado de la institución formalizada. Y, por otro lado, "la educación no formal entra dentro de la profesionalidad de los educadores, de modo que no debe asociarse con la simple improvisación, ni para su ejecución basta la buena voluntad o el compromiso social de sus actores (Sarramona, Vázquez y Colom, 1998, p. 7). Se requiere, en definitiva, la existencia de metas formativas hacia las que orientar la actividad educativa.

No podemos olvidar la necesidad de evaluación en la educación no formal, que llevada a cabo con rigor, nos permita conocer lo realizado para mejorar los procesos y los resultados. Esta característica, aunque no queda reflejada de modo expreso en las definiciones analizadas, subyace al planteamiento general delimitado del concepto de educación no formal.

\section{APORTACIÓN DESDE LA TEORÍA GENERAL DE SISTEMAS}

No queremos que los árboles nos impidan ver el bosque; los árboles son tantos tipos de educación: educación formal, educación informal, educación no formal. Todos ellos constituyen un todo que es la educación y el concepto de educación que sustentan estas páginas es la persona. La persona real de la educación no es el hombre abstracto, sino cada uno de los hombres concretos, desde su propia y específica identidad, y desde las características y posibilidades que se dan en una situación social y cultural determinada. Aunque no queremos extendernos en la consideración sobre el sujeto de la educación, tampoco podemos obviarlo, por eso resumimos nuestra visión personal de la misma a la luz de la síntesis presentada por Medina Rubio (1992), destacando estas notas de la persona como sujeto de educación: "la persona humana es singular, autónoma, es apertura. La persona por naturaleza es un ser abierto a los demás, a la realidad y al mundo que le rodea, es unitaria en la multiplicidad de elementos" (pp. 41-45).

Este enfoque sistémico nos ofrece, pues, el análisis de la educación como una interacción continua y fluida entre el medio, la genética, la cultura, etc., contribuyendo así al desarrollo perfectivo y optimizante de la persona. La educación se da dentro del sistema de la VIDA. La educación es un sistema dentro de otro sistema, que a su vez está dentro de otro y así sucesivamente. "Lo que no podemos olvidar es que se trata de sistemas más amplios, que recogen a otros menos amplios" (Martínez Santos, 1989, p. 77).

Al estudiar la documentación sobre educación no formal, no hemos encontrado reflexiones sobre la misma desde el punto de vista de la Teoría General de Sistemas. Y es curioso, porque la característica de sistemática aparece en varias 
definiciones ${ }^{1}$ y en la investigación educativa actual se ve muy útil el empleo del enfoque sistémico como necesidad de estudiar la educación en el "entorno total" (Casanova y Berlines, 1997, p. 72).

A partir de esta reflexión inicial, descubrimos la necesidad de realizar un análisis de tal teoría que sustente nuestro planteamiento teórico sobre el tema en cuestión, sin ánimo de ser exhaustivos en el desarrollo de la denominada Teoría General de Sistemas.

Junto al neopositivismo lógico, al razonamiento crítico y demás orientaciones metodológicas postpopperianas de Kuhn, Lakatos y Feyerabend, podemos señalar otras corrientes teórico-metodológicas presentes en este siglo y que inciden muy claramente en la investigación educativa actual; y una de ellas es la Teoría General de Sistemas, promovida por Bertalanffy. Para este autor (1980), la Teoría General de Sistemas representa un amplio punto de vista sobre la tecnología moderna, sobre las relaciones entre hombre y máquina, y exige una reorientación de la ciencia y de las disciplinas que va desde la Física y la Biología, a las Ciencias Sociales y del Comportamiento (p. 8).

La Teoría General de Sistemas es un paradigma nuevo en el sentido de paradigma definido por Thomas Kuhn (1982), que supone contemplar la realidad de un modo distinto. Al concepto de paradigma queremos darle el significado que tiene de cambio epistemológico o revolución conceptual y metodológica, con consecuencias trascendentales en la investigación científica y en la vida social y cultural del mundo actual. Se trata de considerar la realidad como "un todo organizado", como una gran organización. La consideración de la realidad como un todo organizado exige el conocimiento, tanto de sus partes u objetos, como de las relaciones existentes entre ellos. Hay que pensar, pues, en términos de sistemas de elementos en interacción mutua. Un sistema es un conjunto de objetos o elementos junto con las relaciones entre los objetos, sus atributos y el entorno que los rodea (Sáez Alonso, 1996, p. 104). O en palabras de Ferrer Figueras (1997) «un sistema es un conjunto de elementos, a, b, c, d, y el conjunto de interrelaciones existentes o de interacciones" (p. 107). El paradigma de la Teoría General de Sistemas descansa en la idea de sistema y se puede encontrar en él una serie de conceptos, procedimientos y modelos de análisis muy útiles en su aplicación a la investigación educativa.

Este planteamiento del estudio de los fenómenos en términos de sistemas contrasta con el enfoque de la ciencia moderna, particularmente la Física, donde se habla del análisis de los fenómenos llevado hasta la determinación de elementos simples y aislables. Considerar la realidad en términos de sistemas requiere,

1. Conviene añadir, a este respecto, que existe en el Plan de Estudios conducentes al Título de Diplomado en Educación Social de la Universidad Complutense de Madrid, una materia optativa denominada Sistemas de Educación no Formal, vinculada al área de conocimiento de Teoría e Historia de la Educación, con cuatro créditos totales (tres teóricos y uno práctico), cuya breve descripción del contenido dice asi: “El sistema de educación no formal. Marco social de la educación no formal. Planificación y programas de educación no formal. Estrategias metodológicas de la educación no formal. Evaluación de programas" (BOE 19 de enero de 1996). El concepto sistema aparece, pues, en el mismo título de la asignatura. 
por tanto, un nuevo método de investigación. El propósito del método de los sistemas es tener en cuenta todas las interacciones entre los elementos de un sistema cuya estructura se pretende estudiar. El análisis de sistemas trata de determinar su estructura interna, es decir, la índole de los elementos que la componen y el tipo o variedad de las relaciones que se establecen entre ellos. "Ludwig V. Bertalanffy no trata a los organismos vivientes como un conjunto de componentes y un conjunto de relaciones entre ellos, sino que los describe como un todo, es decir, como un sistema (Ferrer Figueras, 1997, p. 141).

Estas ideas se pueden trasladar también al campo de la educación. Educar y educación son "un todo organizado" que exige el conocimiento tanto de sus partes y elementos, como de las relaciones existentes entre ellos. La educación es un sistema de elementos en interacción mutua y continua. Desde la Teoría de los Sistemas, esa interacción mutua y continua se identifica con el concepto de relación, que se erige en el punto de partida y en la columna vertebral de todo intercambio personal y social. La comunicación es la base y el fundamento sobre los que se articulan los diferentes tipos de relación que pueden existir, siendo ésta una característica fundamental en los sistemas abiertos. La educación y, en concreto, la educación no formal son, ambas, sistemas abiertos ${ }^{2}$.

La educación no formal es un sistema abierto y genera una serie de procesos de comunicación para lograr un cambio personal y un cambio en el entorno. La educación no formal, como la educación, es un sistema abierto en el cual y a través de la interacción entre sus elementos, se consigue pasar de una situación a otra mejor, de lo que es a lo que debe ser.

Desde esta perspectiva, y según hemos afirmado anteriormente, queremos presentar la educación no formal dentro de la metodología sistémica. La educación es un sistema abierto dentro de otros sistemas y la educación no formal se beneficia de ese concepto de sistema abierto, pues conduce a una idea-eje o ideafuerza de interacción. Interacción entre el organismo, la persona y su entorno o elementos del entorno. La educación no formal conduce a pensar en una relación de la persona con un entorno múltiple y variado, donde no existen fronteras aislantes, sino flexibles y fluidas, entre la persona y el exterior a ella. La educación es, en definitiva, un proceso abierto, en movimiento, donde la educación no formal es un elemento más del desarrollo de la persona.

El método sistémico nos permite superar los límites entre educación y educación no formal y nos ayuda a descifrar la complejidad de la educación y sus procesos de interacción entre los diversos sistemas educativos; evitando yuxtaposiciones, oposiciones o aparentes conflictos de hechos, elementos o estructuras con otros hechos, elementos o estructuras.

Por consiguiente, hablar de educación no formal y educación formal tan rígidamente separados puede llevar a olvidar el planteamiento de la educación como

2. Conviene precisar, por su importancia, que los sistemas se pueden clasificar en sistemas abiertos y sistemas cerrados. Los primeros se relacionan con otros sistemas, intercambian y comunican "materias, energía e información con su medio ambiente" (FERRER FigUERAs, 1997, p. 141). "Todos los sistemas vivientes son sistemas abiertos. Los sistemas no vivientes son cerrados" (AUDIRAC CAMARENA, 1994, p. 58). 
un conjunto de elementos, junto con sus relaciones entre éstos y sus atributos. No podemos dividir la educación en "guetos estancos y estancados de índole intelectual» (Savater, 1997, p. 163). Lo que se oferta sobre educación y educación no formal parece confirmar esta forma de pensar.

El propósito de analizar la educación insistiendo en que es un sistema, invita a tener en cuenta todas las interacciones educativas cuya estructura y dinamismo se pretende estudiar. La vida -el gran sistema- es el medio en el cual se educa. La vida, la economía, la política, la ciencia, el arte, son el medio donde la persona se va educando, donde va viviendo todas las relaciones que se establecen en la educación. Es la totalidad de la vida la que va generando el proceso de educación. Lo contrario es que cada elemento o ámbito se constituya en el medio para educar: la música para la educación musical, el consumo para la educación para el consumo, el clima de paz como medio para la educación pacifista, la ciudad como medio para la educación cívica. De ahí la necesidad de incidir en el desarrollo de la persona por encima de la tipología de acciones y programas educativos. El punto de referencia es la PERSONA. La educación descansa en la persona. El centro, el punto de interés está en la persona más que en las instituciones educativas o en los múltiples aspectos que constituyen lo educativo. Lo cual no es óbice para programar ámbitos de actuación educativa dirigidos a desarrollar las múltiples variables de la EDUCACIÓN. El pensamiento sistémico puede ayudar a afrontar la complejidad de los fenómenos que fecundan la educación actual para luego complementar los de actuación educativa que prosigue después, al lado o sin la escuela.

No se trata tanto de dividir y separar la educación cuanto de recuperar el concepto y la realidad del hecho educativo, manejando "la muy compleja y tupida red de interrelaciones" (Trilla, 1993, p. 178), dimensiones, componentes y características estructurales de la educación, que guardan entre sí relaciones sistemáticas.

La educación no es un campo donde se pueden obviar las relaciones entre todos los elementos que la alimentan, como puede hacer la ciencia física o química que analiza los fenómenos hasta la determinación de los elementos más simples y aislables. Por el contrario, la educación es un sistema cuya importancia radica en analizar sus elementos e interacciones. Por tanto, entender la educación como un sistema, en el sentido descrito anteriormente, constituye un intento de analizar el grado de relación entre todos sus elementos.

\section{DEFINICIÓN COMPRENSIVA DE EDUCACIÓN NO FORMAL}

Son mayoría los autores que consideran la educación de la persona como paso de una situación a otra mejor, de lo que es a lo que debe ser. Conciben la educación como facilitadora de perfeccionamiento, de complemento, de formación (García Aretio, 1992, p. 25).

Otras características atribuidas a la educación son: la tarea educativa tiende al desarrollo integral de todas las facultades humanas; la educación conlleva una influencia y una ayuda de unas personas sobre otras, los educandos; tiene un fin concreto que se sustenta en la concepción que se tenga del hombre, del mundo y de la vida. 
Si partimos de las afirmaciones realizadas por Faure en su Informe de 1973, hay que defender una concepción global del desarrollo de la educación para las sociedades contemporáneas, cuya finalidad consiste en asegurar que "todo individuo tenga la posibilidad de aprender durante toda su vida" (Principio 1, p. 265).

Pero, no solamente ese principio llamado de la "educación permanente" constituye la llave maestra de toda la argumentación, sino que, principalmente, se establece como el elemento motor de nuevas estrategias de educación que, aun atribuyéndole a la enseñanza el lugar que le corresponde, han de esforzarse por distribuir las responsabilidades de esa acción educativa entre todas las instituciones sociales, incluso entre aquellas que normalmente no tienen una vocación educativa (Principio 2, pp. 266-267).

Tales estrategias suponen que el desarrollo de un individuo y de un grupo, en nuestra sociedad contemporánea, depende simultáneamente de tres estructuras distintas para su capacitación, como son: la educación informal, la educación no formal y la educación formal.

Subrayamos así la concepción sistémica de la educación y, aunque seamos reiterativos, desde una perspectiva sistémica. La concepción sistémica de la educación supone el estudio general y global de todo fenómeno concurrente en el acto relacional educativo, como ya hemos explicado pormenorizadamente con anterioridad.

No obstante, y ante la manifiesta dificultad de encuadrar de una manera taxativa a los programas, actividades y medios educativos concretos en cada una de estas categorías, La Belle (1976) propone la consideración de estas tres estrategias como modos predominantes o modos de énfasis. Esto significa que una actuación educativa puede estar inscrita particularmente en uno de los tres tipos señalados, acogiendo secundariamente procedimientos y procesos propios de los dos restantes.

Afirmaba Colom en 1982:

El sistema educativo puede definirse como un sistema formado por la interrelación dinámica con capacidad procesual de la totalidad de instituciones, elementos, unidades, complejos y aspectos, como sus manifestaciones y fenomenología, cuya misión o función sea total o parcialmente educativa, entendiendo la educación en su más amplia expresión; o sea, como conjuntos de pautas a transmitir a las nuevas generaciones para que se dé el mantenimiento y el progreso de la vida humana y sociocultural (pp. 141-142)

Si seguimos el modelo de La Belle, y en la línea planteada por Colom, podemos diferenciar entre modos educativos y características educativas, en el que ambos pueden ser formales, no formales e informales. En este contexto, la escuela se situaría en el modo formal de la educación, aunque en ella sea posible simultanear características no formales (actividades extra-curriculares) e igualmente informales (relaciones entre iguales). Por su parte, los programas educativos sistemáticos pero extraescolares (establecidos en el modo no formal), pueden contener elementos propios del área formal (acreditaciones) y procesos informales (la par- 
ticipación en los mismos). Y, finalmente, los medios de comunicación de masas, que son predominantemente informales, suelen acoger espacios prácticamente formales (emisiones radiofónicas para la UNED) y otros no formales (por ejemplo, un curso para aprender inglés).

Esta estructura resulta especialmente adecuada al referirnos a la educación como globalidad, donde caben los tres ámbitos de desarrollo, consecutiva y confluentemente, los cuales mantienen relaciones funcionales de complementariedad (diversificación de la actividad), suplencia (asunción de tareas emanadas de otro ámbito de desarrollo), sustitución (adquisición de competencias propias de otro campo de acción), refuerzo y colaboración (actuación interdependiente) e interferencia (contradicciones entre el orden de aplicación de los tipos de educación), como las más significativas, cuya primacía se encuentra mediatizada por el entorno, las características de los destinatarios, los objetivos programados, etc. (Fermoso, 1994, pp. 121-122).

Sin embargo, es necesario delimitar por separado sus peculiaridades más relevantes, porque siempre prevalecerá, dependiendo del contexto y el tipo de actividad, un modelo u otro sobre los demás.

En este sentido, la formalidad de un hecho educativo tiene grados de mínima y máxima informalidad en función del factor "intencionalidad educativa" y de "Consciencia educativa". Desde esta perspectiva y como extremos clarificadores, si la persona encargada de la educación, como agente educativo, es consciente de su acción y el receptor de la misma no tiene consciencia de ello, la informalidad es mínima. Si el que educa no tiene consciencia de que lo que está haciendo tiene una intencionalidad formativa y el que recibe esta acción tampoco es consciente de que está siendo educado, la informalidad es máxima -extremos entre los que se incluyen grados intermedios de informalidad (Tarrio, 1993, pp. 157-182)-. Es decir, la intencionalidad (propósito de conseguir objetivos educativos precisos) y la consciencia (constancia patente de la acción a realizar), esencialmente del agente, se constituyen en dos elementos fundamentales alrededor de los cuales resulta posible determinar el nivel de informalidad existente en un hecho educativo formal (Fernández-Salinero, 1995, pp. 39-45).

En este contexto, se establece como consideración desiderativa de complementariedad de actuaciones dentro de estos tres ámbitos de desarrollo (formal, no formal e informal), la "voluntad de configurar un sistema, que facilite al máximo el que cada individuo pueda trazarse su propio itinerario educativo de acuerdo con su situación, necesidades e intereses. Para ello, el sistema ha de ser abierto, flexible, evolutivo, rico en cantidad y diversidad de ofertas y medios educativos" (Trilla, 1992, p. 28)

De la reflexión previa sobre la educación formal, no formal e informal, se deducen los aspectos identificativos de las tres realidades educativas diferenciales y complementarias, precisados en relación a los criterios de sistematicidad e intencionalidad de los aprendizajes y en lo referente a las características de organización, racionalidad y mediación pedagógica de cada uno de los ámbitos señalados.

Esta globalidad en los planteamientos conceptuales se encuentra mediatizada por la diversidad de instancias de donde emanan, e implica que toda actividad 
educativa debe inscribirse en un sistema que comprenda la EDUCACIón total de la PERSONA. Desde la Teoría General de Sistemas donde la base es el sistema, algunos contenidos de la educación formal, de la educación no formal y de la educación informal se trasvasan y otros se funden entre sí, aunque muchas veces cada sistema de educación configure sus propios y diferentes escenarios donde se representan iguales contenidos pero para audiencias distintas.

Por lo tanto, una definición comprensiva del concepto de educación no formal nos invita a contemplarla dentro de esta estructura sistémica, superando planteamientos más lineales como los establecidos por Touriñán en los años ochenta, en los que se descubre una aproximación entre la educación formal y no formal, enfrentadas abiertamente con la educación informal (ver cuadro 2).

\begin{tabular}{|l|l|}
\hline $\begin{array}{l}\text { Educación } \\
\text { formal }\end{array}$ & $\begin{array}{l}\text { Educación } \\
\text { informal }\end{array}$ \\
\hline $\begin{array}{l}\text { Educación } \\
\text { no formal }\end{array}$ & \\
\hline
\end{tabular}

CUADro 2. Touriñán, 1984, p. 122

Esta aproximación entre educación formal y no formal se debe a que poseen entre sí un atributo común que no comparten con la educación informal: el de la organización y sistematización intencionales derivadas de estímulos directamente educativos. "Son dos especies, de las cuales, una está representada a su vez, por dos subespecies" (Touriñán, 1984, p. 121).

Presentar la educación no formal según el cuadro 2 parece que es representar o sectorizar la educación por su naturaleza, es decir, considerar a la educación no formal como aquellas acciones o programas que no encuentran acomodo en el ámbito escolar administrativo, institucional, formal, graduado, jerarquizado; confundiendo así educación con una parte de ella que es la escolarización y el aprendizaje y arrojando la educación informal al reino de lo extraescolar. Se subrayan o delimitan así los diversos tipos de educación por criterios metodológicos y estructurales. Y ¿dónde queda la interacción que se da en las actividades educativas, pertenezcan o no al sistema escolar, entre persona/educación/alumno/conocimientos? ¿Acaso va a ser más educativo un proceso, competencia, destreza, actitud o conocimiento por darse en la educación primaria que por darse en una educación no conformada o reglada por la legislación educativa?

El presente esquema, además, no resulta del todo exacto en sus formulaciones. Existen ejemplos de educación informal, como la actividad educativa desarrollada a partir de los medios de comunicación de masas que contiene un relevante componente sistémico; ni resuelve tampoco los problemas implícitos en la relación entre los tres conceptos (Fernández-Salinero, 1995, p. 43). 
Precisamente, para intentar solventar las dificultades emanadas de los planteamientos precedentes, se comienza a vislumbrar una nueva concepción de la educación no formal dentro de una estructura circular, donde este ámbito educativo junto con el formal y el informal se convierte en un elemento más del denominado y, actualmente muy desarrollado, énfasis en la educación permanente.

Una perspectiva en alza en el momento actual es la propugnada por Vázquez (1998). Este autor comienza a hablar de un enfoque sistémico de la educación regulado por una estructura normativa que identifica como eje central de desarrollo el concepto de la educación permanente (ver cuadro 3 ).

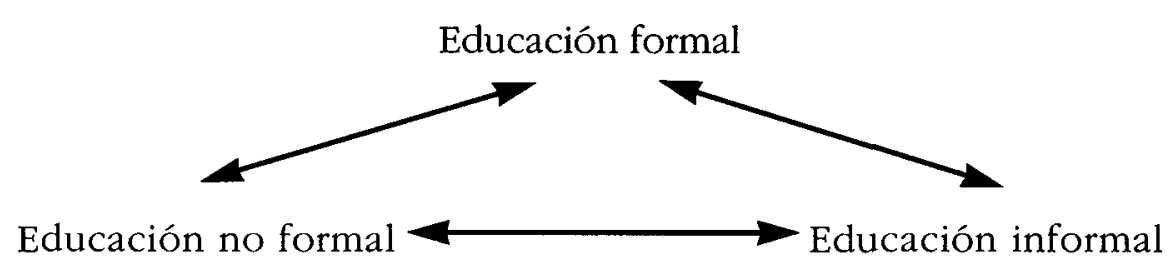

CUADRo 3. Vázquez, 1998, p. 17

La idea que subyace a este enfoque es la de que la educación permanente debe considerarse como un principio de un sistema general de educación, cuya meta no debe ponerse en una prolongación de la escolaridad obligatoria, sino en la prestación de una educación básica de calidad y universal que asegure la educación y la autoformación permanentes (Vázquez, 1998, p. 18).

Diferente del cuadro 2 es el cuadro 3. No hay muros rígidos entre las distintas formas de educación. Se da más bien una dinámica, un proceso, una interacción entre los tres tipos de educación, pero no se declara fundamental la relación sistémica entre ellas. Los procesos se relacionan entre sí y generan aprendizajes y efectos educativos distintos y, por la imagen del cuadro, distantes, diferenciados y específicos. La forma de triángulo, no obstante, puede generar equívocos, aunque sus vértices mantienen entre sí una relación explícita y complementaria.

Ambas concepciones, la de Touriñán y la de Vázquez, implican un sustrato sistémico; sin embargo, mientras la primera lo utiliza para ordenar diferentes tipos de educación, la segunda lo emplea para identificar un planteamiento educativo general y homogéneo en la "aldea global" en la que nos vemos inmersos.

Nosotros vamos más allá e intentamos aproximarnos a un concepto de la educación no formal que se inscribe en otro más amplio de EDUCACIón, constituida ésta como un sistema que tiene como eje de desarrollo a la PERSONA; pues sin la persona no hay educación, pero sin educación la persona no puede desarrollar realmente su humanización, individual y socialmente.

Nuestro empeño se traduce en los gráficos siguientes (cuadro 4 y cuadro 5), en los que intentamos explicar nuestra postura desde los planteamientos precedentes derivados de la Teoría General de Sistemas, y donde la educación no formal concretamente se conformaría como aquella educación producida fuera de la 
escuela, pero institucionalizada, sistematizada o metódicamente prefigurada con una intervención pedagógica intencional y consciente.

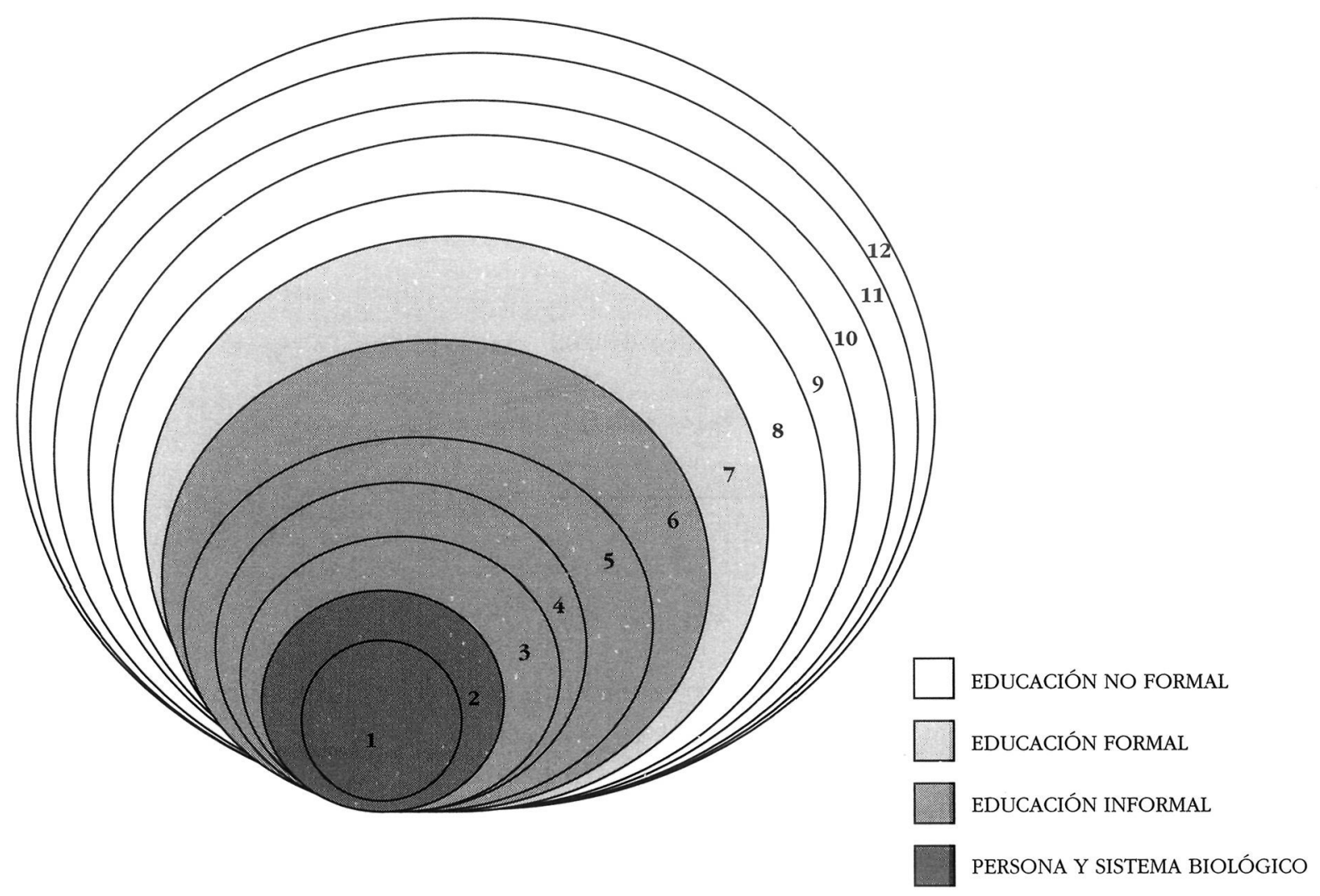

CUADRO 4. Elaboración propia

En este cuadro 4 empleamos la línea espiral y las diferentes gamas de color, desde el blanco hasta el gris oscuro, para manifestar de modo visual un punto de encuentro que para nosotros es la PERSONA (gris más oscuro). Se matiza la gradación de los colores en función de dos criterios: el primero, la proximidad o lejanía de los planteamientos educativos a ese centro o eje de desarrollo que es la persona. El segundo, la numeración de cada espiral para identificar los diversos sistemas de educación no formal, educación formal y educación informal.

Y en el cuadro 5 otorgamos un significado preciso a cada color y cada número establecidos en el cuadro anterior.

La educación desde la Teoría General de Sistemas incluye diferentes ámbitos para su desarrollo. Es decir, implica a diferentes organizaciones, instituciones y participantes en su estructura sistémica. Todos estos agentes son importantes en cada estructura, para instruir y enseñar, para formar y educar, pero no son los únicos.

El cuadro 4 intenta apoyar esta idea gráficamente. En él vemos cómo la persona es el eje del desarrollo del sistema educación y punto de confluencia de todos los demás sistemas. 


\begin{tabular}{|rl|}
\hline 1. & PERSONA \\
2. & SISTEMA BIOLÓGICO \\
\hline 3. & SISTEMA FAMILIAR \\
4. & SISTEMA LÚDICO \\
5. & SISTEMA CULTURAL \\
6. & SISTEMA SOCIAL \\
\hline 7. & SISTEMA EDUCATIVO/ESCOLAR \\
\hline 8. & SISTEMA SANITARIO \\
9. & SISTEMA LABORAL/PROFESIONAL \\
10. & SISTEMA ECONÓMICO \\
11. & SISTEMA IDEOLÓGICO \\
12. & SISTEMA POLÍTICO \\
\hline
\end{tabular}

CUADRO 5. Elaboración propia

La persona está en una interacción continua y fluida. La persona es abrazada por todo tipo de educación y desarrolla todo su potencial cultural y social a través de la educación no formal, formal e informal, constituyéndose en el punto de encuentro de todas ellas. Aunque cada sistema de educación optimice algún ámbito concreto de la persona, ésta es, en su totalidad, centro y meta de la multiplicidad de elementos que la componen.

En el mencionado cuadro 4 se visualizan más claramente estas ideas. En él, los círculos 1 y 2 se identifican con los sistemas de la persona y su sistema biológico, contactando entre sí y con todos los demás círculos en espiral; del 3 al 6 , se encuentran los sistemas de la educación informal; el 7 se refiere a la educación formal, y del 8 al 12 a la educación no formal.

Vemos, pues, en este cuadro que la educación aparece como un todo organizado, observándose a la vez todas sus partes y sus relaciones, no como compartimentos estancos, sino más bien como sistemas interconectados y distribuidos en derredor de la persona, eje y punto de apoyo de la educación.

La proximidad o lejanía de esos sistemas de la persona nos indica las etapas evolutivas del sistema educación, en el cual encontramos al sistema de la educación no formal favoreciendo el crecimiento de la persona desde diferentes áreas y distintas realidades, apoyándose en organizaciones y puntos de encuentro alternativos y facilitando un armónico perfeccionamiento de las posibilidades individuales y sociales de la persona.

No obstante, el punto de confluencia de la estructura planteada nos está indicando dos cosas concretamente. 
La primera, que cada elemento del sistema educación por el que la persona va pasando, y en el que se va formando, mantiene una relación de proximidad con la misma, en ocasiones por la propia iniciativa individual y, en otras, por la influencia del ambiente o del entorno en el que se desarrolla.

La segunda, que la proximidad de los sistemas nos indica la interrelación entre los mismos y entre éstos y la persona, de manera constante, existiendo una mayor relación entre cada uno de esos sistemas y la persona en función del momento evolutivo por el que ésta esté pasando.

Ambas ideas apoyan nuestra concepción sistémica de la educación no formal, la cual es considerada como aquella educación que convive con la persona, ayudándola a desarrollarse fundamentalmente en aquellas etapas de crecimiento en las que la educación formal deja un hueco para la decisión personal y la educación informal se encuentra situada en un segundo plano de ayuda.

Esto significa que en los momentos decisivos del perfeccionamiento humano la educación no formal adquiere protagonismo, aunque siga siendo un sistema más de los múltiples que conforman el concepto global de educación.

Nos referimos en concreto al sistema sanitario, al sistema laboral/profesional, al sistema económico, al sistema ideológico o al sistema político como los más relevantes y que se incluyen dentro del sistema de la educación no formal.

Estos sistemas proporcionan a la persona las herramientas para el triunfo y requieren una estructurada y sistémica organización de sus postulados, superando los planteamientos del sistema escolar y completando las perspectivas arraigadas en el sistema familiar, lúdico, cultural o social.

\section{A MODO DE CONCLUSIÓN}

El análisis de la educación no formal desde la Teoría General de Sistemas que hemos desarrollado a través de estas páginas, no nos permite hablar de fusión de los distintos tipos de educación, sino de interrelación entre ellos, pues la fusión haría resentirse la estructura educativa.

La reflexión sobre la educación no formal desde la Teoría General de Sistemas insiste en la educación como sistema y en el sistema persona/alumno/adulto. Y en este sistema participa toda la educación/persona/alumno/familia/sociedad/ administración. De tal modo que no sólo hay que considerar los elementos diferentes sino también lo que sostiene/une/interrelaciona a todos ellos. Los campos de cada educación son teóricamente distintos, pero en la vida van muchas veces unidos, otras se complementan y las más de las veces se prolongan los unos a los otros en ámbitos, objetivos, contenidos y métodos distintos, que no distantes. La educación es algo global, de una complejidad extraordinaria por los elementos del sistema que la constituyen y por los actores que en ella intervienen.

La educación se da en situaciones de interacción y de reciprocidad-como se refleja en nuestro estudio-, de manera que la persona educada es el resultado de un proceso sistémico donde todos los elementos actúan implícita o explícitamente, de modo natural o de modo sistematizado, pero siempre dentro de una relación entre seres humanos. 
Esta perspectiva nos permite identificar a la educación no formal como un sistema más, pero aquel que ayuda a la persona a desarrollar las partes de su personalidad nacidas en el seno del sistema de la educación informal (familia, cultura, sociedad), despertadas en el sistema de la educación formal (escuela) y realmente pulidas, orientadas y concretadas gracias al sistema de la educación no formal (sanidad, trabajo, economía, ideas, política), piedra angular para el auténtico y perfectivo desarrollo continuo de la persona.

\section{BIBLIOGRAFÍA}

Ahmed, M. (1989): "Economía de la educación no formal". En Husen, T. y Postlethwaite, T. N. (dirs.): Enciclopedia Internacional de la Educación. Vicens Vives. Barcelona, pp. 1.847-1.849.

AHMED, M. y CoOmBs, Ph. (1975): La lucha contra la pobreza rural. El aporte de la educación no formal. Tecnos. Madrid.

Aitchison, J. (ed.) (1984): Tesauro de la Unesco, vol. 2. Unesco. Paris.

BERTALANFFY, L. V. (1980): Teoria general de los sistemas. Fundamentos, desarrollo, aplicaciones. Fondo Cultura Económica. Madrid.

- (1981): "Historia y situación de la teoría general de sistemas". En KLIR, G. J. (comp.): Tendencias en la teoria general de sistemas. Alianza. Madrid, pp. 29-53.

CASANOVA, U. y BeRLINER, D. (1997): "La investigación educativa en Estados Unidos: El último cuarto de siglo". Revista de Educación, 312, pp. 43-80.

CAstillejo, J. L. (1987): Pedagogía tecnológica. Ceac. Barcelona.

CoOmBs, Ph. (1989): "Educación no formal: Estudios comparativos". En Husen, T. Y Postlethwaite, T. N. (dirs.): Enciclopedia Internacional de la Educación. Vicens Vives. Barcelona, pp. 1.850-1.852.

Colom, A. J. (1982): Teoría y metateoría de la educación. Trilla. México.

- (1992): “Estrategias metodológicas en la educación no formal”. En SARRAmona, J. (ed.): La educación no formal. Ceac. Barcelona, pp. 51-73.

DeloRs, J. (1996): La educación encierra un tesoro: Informe a la Unesco de la Comisión Internacional sobre la Educación para el siglo XXI. Santillana/Unesco. Madrid.

FAURE, E. ET AL. (1973): Aprender a ser (1 $1^{\mathrm{a}}$ edición). Alianza. Madrid.

Fermoso, P. (1994): Pedagogía social. Fundamentación científica. Herder. Barcelona.

- (1997): “Interculturalismo y educación no formal». En Petrus, A. (coord.): Pedagogía Social. Ariel. Barcelona, pp. 248-267.

FERNÁNDEZ-SALINERO, C. (1995): La Formación en las Pequeñas y Medianas Empresas (PYME): Diagnóstico Estructural y Prospección de Estrategias Tecnológicas Alternativas. Tesis Doctoral Inédita. Madrid.

FERRER Figueras, L. (1997): Del paradigma mecanicista de la ciencia al paradigma sistémico. Universidad de Valencia. Valencia.

Fundación SANTULlana (1991): La educación no formal: una prioridad de futuro. Documentos de un Debate. Madrid.

FURTER, P. (1976): "La formation extraescolaire et le développement dépendant". Cabiers de l'Intitut Universitaire d'Études du Développement, 4.

García Aretio, L. (1992): “Concepto de educación”. En Medina Rubio, R., Rodríguez Neira, T. y García ARETIO, L.: Teoría de la educación, 1, UNED. Madrid, pp. 13-38. 
RAFAEL SÁEZ ALONSO Y CAROLINA FERNÁNDEZ-SALINERO MIGUEL APROXIMACIÓN A UN PLANTEAMIENTO SISTEMÁTICO DE LA EDUCACIÓN...

García Carrasco, J. (1991): La educación básica de adultos. Ceac. Barcelona.

KuHn, T. S. (1982): La estructura de las revoluciones científicas. Fondo de Cultura Económica. México.

LA Belle, T. J. (1981): "An introduction to the nonformal education of children and youth". Comparative Education Review, 25 (3), pp. 313-329.

- (1982): "Formal, nonformal, and informal education: a holistic perspective lifelong learning". International Review of Education, XXVIII, pp. 159-175.

LENGRAND, P. (1973): Introducción a la educación permanente. Teide. Barcelona.

LOWE, J. (1989): "Educación no formal postsecundaria". En HuSEN, T. y POSTLETHWArTe, T. N. (dirs.): Enciclopedia Internacional de la Educación. Vicens Vives. Barcelona, pp. 1.844-1.847.

MaRTínez SANTOS, S. (1989): Estructura curricular y modelos para la innovación. Ediciones Nieva. Madrid.

Medina Rubio, R., Rodríguez Neira, T. y García Aretio, L. (1992): Teoría de la educación, 1. UNED. Madrid.

NASSIF, R. (1983): Teoría de la educación. Problemática pedagógica contemporánea. Cincel. Madrid.

Núñez Cubero, L. (ed). (1993): Metodologias de investigación en la educación no formal. Aportaciones teóricas. Preu Spínola. Sevilla.

Puig, J. M. y Trilla, J. (1987): Pedagogía del ocio. Laertes. Barcelona.

QuinTANa Cabanas, J. Ma (1977): Sociología de la educación. La enseñanza como sistema social. Hispano Europea. Barcelona.

- (coord.) (1991): Iniciativas sociales en educación informal. Rialp. Madrid.

RADCHIFFe, D. J. y COlleta, N. J. (1989): "Educación no formal". En Husen, T. y Postlethwatte, T. N. (dirs.): Enciclopedia Internacional de la Educación. Vicens Vives. Barcelona, pp. 1.837-1.842.

REQUEJO, A. (1994): "La educación no formal de adultos: ciudad educativa y ciudad sana en el contexto del desarrollo comunitario y la intervención en la tercera edad". Teoría de la Educación, VI, pp. 61-76.

SÁEz Alonso, R. (1996): Proyecto Docente presentado para optar a la Plaza de Profesor Titular de Universidad del área de conocimiento "Teoría e Historia de la Educación" con actividades a realizar: Teoría de la Educación. Bases Metodológicas de la Investigación Educativa, en la Universidad Complutense de Madrid.

SANCHO GIL, J. Mª y Hernández Hernández, F. (1997): “La investigación educativa como espacio de dilemas y contradicciones". Revista de Educación, 312, pp. 81-110.

SarRamona, J. (ed.) (1992): La educación no formal. Ceac. Barcelona.

- Vázquez, G. y Ucar, X. (1992): "Evaluación de la educación no formal". En Sarramona, J. (ed.), La educación no formal. Ceac. Barcelona, pp. 91-126.

- VázQuez, G. y Colom, A. J. (1998): Educación no formal. Ariel. Barcelona.

SAVATER, F. (1997): El valor de educar. Ariel. Barcelona.

SRINIVASAN. L. (1989): «Educación no formal: Enseñanza». En Husen, T. y Postlethwaite, T. N. (dirs.): Enciclopedia Internacional de la Educación. Vicens Vives. Barcelona, pp. 1.8521.855 .

TARrio, J. A. (coord.) (1993): La educación y su problemática. O Castro Sada. A Coruña.

TOuRIN̂́n, J. M. (1984): "Análisis teórico del carácter "formal", "no formal" e "informal".. En Aznar, P. y otros: Conceptos y propuestas (I). Papers d'Educació. Nau Llibres. Valencia, pp. 111-113.

TrILla, J. (1986): La educación informal. PPU. Barcelona. 
- (1985): La educación fuera de la escuela. Enseñanza a distancia, por correspondencia, por ordenador, por radio, vídeo y otros medios no formales. Colección Nueva Paideia. Planeta. Barcelona.

- (1992): "La educación no formal. Definición, conceptos básicos y ámbitos de aplicación". En Sarramona, J. (ed.): La educación no formal. Ceac. Barcelona, pp. 9-50.

- (1993 a): Otras educaciones. Animación sociocultural, formación de adultos y ciudad educativa. Anthropos. Barcelona.

- (1993 b): La educación fuera de la escuela. Ámbitos no formales y educación social. Ariel. Barcelona.

VÁzQuez, G. (1995): “Ámbitos y dimensiones de la educación. Los ámbitos de la educación formal y no formal. En BARTOLOMÉ, D. ET AL.: Formación de profesores de educación secundaria. Complutense. Madrid, pp. 31-41. 